\title{
Joint Turbo Equalization for Relaying Schemes over Frequency-Selective Fading Channels
}

\author{
Houda Chafnaji ${ }^{\ddagger *}$, Tarik Ait-Idir ${ }^{\ddagger *}$, Halim Yanikomeroglu ${ }^{+}$, and Samir Saoudi* \\ ${ }^{\ddagger}$ Communications Systems Department, INPT, Madinat Al Irfane, Rabat, Morocco \\ *Signal and Communications Department, INSTITUT TELECOM/ TELECOM Bretagne, CS 83818, 29238 Brest Cedex, France. \\ ${ }^{+}$Broadband Communications and Wireless Systems (BCWS) Centre, \\ Department of Systems and Computer Engineering Carleton University, Ottawa, Canada \\ Emails: houda.chafnaji,samir.saoudi@telecom-bretagne.eu, \\ aitidir@ieee.org, halim@sce.carleton.ca
}

\begin{abstract}
We propose a single carrier joint frequency domain equalization and interference cancellation (FDE-IC) with diversity combining for different relaying schemes. We consider amplify-and-forward (AF) and ACK/NACK-aided decodeand-forward (DF) modes over multiple-input multiple-output (MIMO) frequency selective relay channels. During the first time slot, the source transmits to both the relay and destination. In the second slot, the relay transmits the signal packet to the destination in the case of AF mode, while in DF the data block is transmitted either by the relay or the source depending on the ACK/NACK feedback. By deriving an equivalent source-relay-destination $(S \rightarrow R \rightarrow D)$ channel for the AF mode, we propose an integrated iterative minimum mean square error (MMSE)-FDE-IC-aided transmission combining scheme where reception over multiple slots is viewed as virtual antennas. We also derive the joint combining scheme in the case of ACK/NACK-aided DF. Block error rate (BLER) performance is evaluated for different system settings.
\end{abstract}

\section{Categories and Subject Descriptors}

C.2.1 [Network Architecture and Design]: Wireless communication.

\section{General Terms}

Algorithms, Performance.

\section{Keywords}

Cooperative relaying, multiple-antenna systems, joint equalization and interference cancellation.

\section{INTRODUCTION}

Permission to make digital or hard copies of all or part of this work for personal or classroom use is granted without fee provided that copies are not made or distributed for profit or commercial advantage and that copies bear this notice and the full citation on the first page. To copy otherwise, to republish, to post on servers or to redistribute to lists, requires prior specific permission and/or a fee.

IWCMC'09, June 21-24, 2009, Leipzig, Germany

Copyright (C) 2009 ACM 978-1-60558-569-7/09/06 ...\$5.00.
Relaying is an efficient diversity technique that allows to combat multi-path fading in wireless communications [1-3]. In a relay system, one or more relays assist the communication between the source and destination to form a multipleinput multiple-output (MIMO) system array and exploit space-time diversity. Several interesting relaying schemes have been proposed, among which are two basic modes: amplify-and-forward (AF) and decode-and-forward (DF).

The AF strategy represents the simplest way that a relay may cooperate with the source and destination. Under this scheme, the relay simply amplifies the received signal and forwards it towards destination. However, in the DF mode, the relay first decodes the signal received from the source, reencodes and retransmits it to the destination. This approach suffers from error propagation when the relay transmits an erroneously decoded data block $[2,4]$. Selective protocols, where the relay transmits only when it can reliably decode the data packet, have been introduced as an effective method to reduce error propagation [5-7]. In [8], the authors proposed a ACK/NACK-aided selective DF protocol where the relay transmits only if it correctly decodes the packet, otherwise, it sends back a NACK message to the source that directly transmits the frame to the destination. In this paper, we refer to this scheme as ACK/NACK-aided DF.

To improve spatial diversity of a relay system, signals received over the source-destination and relay-destination links are combined at the receiver side. Most of the research work in this area focused on flat fading channels. However, in practical systems, channels connecting the source, relay and destination may suffer from inter-symbol interference (ISI) caused by frequency-selective fading. Block equalization was proposed in [9-11] for the so-called protocol III [12] where the source sends to the relay during the first slot and both the source and relay send to the destination in the second slot. In [13], the authors introduced a maximum ratio combining (MRC)-aided strategy for protocol I [12], i.e., a scheme similar to protocol III except that the destination receives direct signal from the source in the first slot, under the AF mode and operating over a frequency-selective relay channel.

In this paper, we consider a single carrier (SC) broadband coded relay system where the source, relay, and destination are equipped with multiple antennas. The system is supposed to operate under the so-called protocol II [12], where the source sends to both the relay and destination in the first slot, and the relay sends to the destination in the sec- 
ond slot. This protocol scheme presents a generalization of automatic repeat request (ARQ) mechanisms. We consider both $\mathrm{AF}$ and DF modes. In the case of AF, the system elements communicate using protocol II, while in the case of $\mathrm{DF}$, either the source or relay transmit to the destination depending on the ACK/NACK feedback from the relay, i.e., ACK/NACK-aided DF. We focus on joint iterative (turbo) frequency domain equalization and interference cancellation (FDE-IC) with diversity combining at the destination. We propose an integrated iterative minimum mean square error (MMSE)-FDE-IC-aided transmission combining strategy for both $\mathrm{AF}$ and $\mathrm{DF}$ modes, where multiple slot receptions are viewed as virtual antennas. Performance of the proposed combining scheme is evaluated via computer simulations for many relay configurations.

The remainder of the paper is organized as follows: Section ?? presents the considered relaying schemes and their corresponding communication models. Section ?? details the proposed turbo equalization-aided combining strategy. Numerical results are provided in Section ??. The paper is concluded in Section ??.

\section{Notations}

- $\operatorname{diag}\{\mathbf{x}\}$ and $\operatorname{diag}\left\{\mathbf{X}_{1}, \cdots, \mathbf{X}_{m}\right\}$ denote the diagonal matrix and the block diagonal matrix constructed from $\mathbf{x} \in \mathbb{C}^{n}$ and from $\mathbf{X}_{1}, \cdots, \mathbf{X}_{m} \in \mathbb{C}^{n_{1} \times n_{2}}$, respectively.

- $(.)^{\top}$ and $(.)^{\mathrm{H}}$ are the transpose and the transpose conjugate of the argument, respectively.

- $\mathbf{U}_{T}$ is a unitary $T \times T$ matrix whose $(m, n)$ th element is $\left(\mathbf{U}_{T}\right)_{m, n}=\frac{1}{\sqrt{T}} e^{-j(2 \pi m n / T)}$, and $j=\sqrt{-1}$.

- $\mathbf{U}_{T, N}=\mathbf{U}_{T} \otimes \mathbf{I}_{N}$, where $\mathbf{I}_{N}$ is the $N \times N$ identity matrix and $\otimes$ denotes the Kronecker product.

- For $\mathbf{x} \in \mathbb{C}^{T N}, \mathbf{x}_{f}$ denotes the Fourier transform of $\mathbf{x}$, i.e., $\mathbf{x}_{f}=\mathbf{U}_{T, N} \mathbf{x}$.

\section{RELAYING SCHEMES AND COMMUNI- CATION MODELS}

\subsection{Relay System}

We consider a system with $M$ antenna source, $N$ antenna destination and $K$ antenna relay to assist the communication between the source and the destination, where $N \geq K \geq M$. The source-relay $(S \rightarrow R)$, source-destination $(S \rightarrow D)$, and relay-destination $(R \rightarrow D)$ channels are frequency selective and have $L_{S R}, L_{S D}$, and $L_{R D}$ symbolspaced taps, respectively. Cyclic prefix-aided transmission is assumed. The channel matrices corresponding to the three links are $\mathbf{H}_{0}^{(S R)}, \cdots, \mathbf{H}_{L_{S R}-1}^{(S R)} \in \mathbb{C}^{K \times M}, \mathbf{H}_{0}^{(S D)}, \cdots, \mathbf{H}_{L_{S D}-1}^{(S D)} \in$ $\mathbb{C}^{N \times M}$, and $\mathbf{H}_{0}^{(R D)}, \cdots, \mathbf{H}_{L_{R D}-1}^{(R D)} \in \mathbb{C}^{N \times K}$. Their entries are zero-mean circularly symmetric complex Gaussian and satisfy the following normalizations,

$$
\left\{\begin{array}{l}
\mathbb{E}\left[\operatorname { d i a g } \left\{\sum_{l=0}^{L_{S R}-1} \mathbf{H}_{l}^{(S R)} \mathbf{H}_{l}^{\left.\left.(S R)^{\mathrm{H}}\right\}\right]=}=M \mathbf{I}_{K},\right.\right. \\
\mathbb{E}\left[\operatorname{diag}\left\{\sum_{l=0}^{L_{S D}^{-1}} \mathbf{H}_{l}^{(S D)} \mathbf{H}_{l}^{\left.\left.(S D)^{\mathrm{H}}\right\}\right]=}\right\} \mathbf{I}_{N},\right. \\
\mathbb{E}\left[\operatorname{diag}\left\{\sum_{l=0}^{L_{R D}^{-1}} \mathbf{H}_{l}^{(R D)} \mathbf{H}_{l}^{(R D)^{\mathrm{H}}}\right\}\right]=K \mathbf{I}_{N} .
\end{array}\right.
$$

The average energies of the three links are $E_{S R}, E_{S D}$ and $E_{R D}$, and take into account the path-loss and shadowing effects of each link.

We consider two relaying schemes: i) amplify-and-forward (AF), and ii) ACK/NACK-aided decode-and-forward (DF). Both schemes use two time slots for sending one information block from the source to the destination, where each slot spans $T$ channel uses (c.u). First, the source encodes its data block using a space-time bit interleaved coded modulation (STBICM) encoder. The resulting symbol vector is given by,

$$
\mathbf{s}=\left[\mathbf{s}_{0}^{\top}, \cdots, \mathbf{s}_{T-1}^{\top}\right]^{\top} \in \mathcal{S}^{T M},
$$

where $\mathbf{s}_{i} \in \mathcal{S}^{M}$ is the $M \times 1$ symbol vector at c.u $i=$ $0, \cdots, T-1$, and $\mathcal{S}$ is the symbol constellation. During the first slot, the source inserts a cyclic prefix (CP) symbol word of length $T_{C P}^{S} \rightarrow^{D} \geq \max \left(L_{S R}, L_{S D}\right)$, then transmits the resulting symbol frame to both the relay and destination. After $\mathrm{CP}$ deletion, the $N \times 1$ signal obtained at the destination is,

$$
\mathbf{y}_{i}^{(1)}=\sqrt{E_{S D}} \sum_{l=0}^{L_{S D}-1} \mathbf{H}_{l}^{(S D)} \mathbf{s}_{(i-l) \bmod T}+\mathbf{n}_{i}^{(1)},
$$

where $\mathbf{n}_{i}^{(1)} \sim \mathcal{N}\left(\mathbf{0}_{N \times 1}, \sigma^{2} \mathbf{I}_{N}\right)$ is the thermal noise. During the second slot, the transmission strategy depends on the considered relaying scheme. In the case of $\mathrm{AF}$ mode, the relay amplifies and sends the block of received signals to the destination. When ACK/NACK-aided DF is considered, the symbol packet is sent to the destination either by the source or the relay depending on the ACK/NACK feedback. In other words, the relay sends back a NACK message to the destination if the decoding outcome at the relay is erroneous then the source directly sends the symbol frame to the destination, while the relay transmits the symbol frame to the destination if it correctly decodes the packet. In the following, we provide communication models for both relaying schemes.

\subsection{AF Scheme}

After $\mathrm{CP}$ removal at the relay side, the $K \times 1$ received signal is expressed as,

$$
\mathbf{y}_{i}^{(S R)}=\sqrt{E_{S R}} \sum_{l=0}^{L_{S R}-1} \mathbf{H}_{l}^{(S R)} \mathbf{s}_{(i-l) \bmod T}+\mathbf{n}_{i}^{(S R)},
$$

where $\mathbf{n}_{i}^{(S R)} \sim \mathcal{N}\left(\mathbf{0}_{K \times 1}, \sigma^{2} \mathbf{I}_{K}\right)$ is the thermal noise at the relay. During the second time slot, the relay first normalizes received signals $\mathbf{y}_{i}^{(S R)}$ as,

$$
\tilde{\mathbf{y}}_{i}^{(S R)}=\frac{1}{\sqrt{M E_{S R}+\sigma^{2}}} \mathbf{y}_{i}^{(S R)},
$$

then inserts a CP signal word of length $T_{C P}^{R \rightarrow D} \geq L_{R D}$ and transmits the resulting signal packet to the destination. After CP deletion, the $N \times 1$ received signal during the second slot is

$$
\begin{aligned}
\mathbf{y}_{i}^{(R D)}=\sqrt{\frac{E_{R D} E_{S R}}{M E_{S R}+\sigma^{2}}} \sum_{l=0}^{L_{S R D}-1} \mathbf{H}_{l}^{(S R D)} \mathbf{s}_{(i-l) \bmod T}+ \\
\widetilde{\mathbf{n}}_{i}^{(S R D)}
\end{aligned}
$$


where

$$
\widetilde{\mathbf{n}}_{i}^{(S R D)}=\sqrt{\frac{E_{R D}}{M E_{S R}+\sigma^{2}}} \sum_{l=0}^{L_{R D}-1} \mathbf{H}_{l}^{(R D)} \mathbf{n}_{(i-l) \bmod T}^{(S R)}+\mathbf{n}_{i}^{(R D)}
$$

is the effective zero-mean Gaussian noise at the destination side and has conditional covariance matrix,

$\Theta_{\mid \mathbf{H}^{(R D)}}=\sigma^{2}\left(\mathbf{I}_{N}+\frac{E_{R D}}{M E_{S R}+\sigma^{2}} \sum_{l=0}^{L_{R D^{-1}}} \mathbf{H}_{l}^{(R D)} \mathbf{H}_{l}^{(R D)^{H}}\right)$.

$\mathbf{H}_{0}^{(S R D)}, \cdots, \mathbf{H}_{L_{S R D}-1}^{(S R D)} \in \mathbb{C}^{N \times M}$ is the equivalent multipath channel corresponding to $\operatorname{link} S \rightarrow R \rightarrow D$, and has $L_{S R D}=$ $L_{S R}+L_{R D}-1$ symbol-spaced taps. The $l$ th equivalent tap channel matrix is expressed as,

$$
\begin{gathered}
\mathbf{H}_{l}^{(S R D)}=\sum_{n=\max \left(0, l-L_{S R}+1\right)}^{\min \left(l, L_{R D}-1\right)} \mathbf{H}_{n}^{(R D)} \mathbf{H}_{l-n}^{(S R)}, \text { if } L_{R D} \geq L_{S R} \\
\mathbf{H}_{l}^{(S R D)}=\sum_{n=\max \left(0, l-L_{R D}+1\right)}^{\min \left(l, L_{S R}-1\right)} \mathbf{H}_{l-n}^{(R D)} \mathbf{H}_{n}^{(S R)}, \text { Otherwise. }
\end{gathered}
$$

To perform joint equalization over signal packets received over links $S \rightarrow D$ and $R \rightarrow D$, the destination proceeds by a whitening of the effictive noise using the Cholesky Factorization of $\frac{1}{\sigma^{2}} \boldsymbol{\Theta}_{\mid \mathbf{H}^{(R D)}}=\mathbf{L} \mathbf{L}^{H}$, where $\mathbf{L}$ is a $N \times N$ lower triangular matrix. This yields signal vector,

$$
\begin{aligned}
\mathbf{y}_{i}^{(2)} & =\mathbf{L}^{-1} \mathbf{y}_{i}^{(R D)} \\
& =\sqrt{E_{2}} \sum_{l=0}^{L_{2}-1} \mathbf{H}_{l}^{(2)} \mathbf{s}_{(i-l) \bmod T}+\mathbf{n}_{i}^{(2)},
\end{aligned}
$$

where

$$
\begin{cases}\mathbf{H}_{l}^{(2)} & =\mathbf{L}^{-1} \mathbf{H}_{l}^{(S R D)} \\ L_{2} & =L_{S R D} \\ E_{2} & =\frac{E_{R D} E_{S R}}{M E_{S R}+\sigma^{2}}\end{cases}
$$

and $\mathbf{n}_{i}^{(2)} \sim \mathcal{N}\left(\mathbf{0}_{N \times 1}, \sigma^{2} \mathbf{I}_{N}\right)$ is the whitened effictive noise at the destination side.

\subsection{ACK/NACK-Aided DF Scheme}

After CP deletion, the $N \times 1$ received signal at the destination side during the second slot is expressed as,

$$
\mathbf{y}_{i}^{(2)}=\sqrt{E_{2}} \sum_{l=0}^{L_{2}-1} \mathbf{H}_{l}^{(2)} \mathbf{s}_{(i-l) \bmod T}+\mathbf{n}_{i}^{(2)}
$$

where

$$
\begin{cases}L_{2}=L_{R D}, E_{2}=E_{R D}, \mathbf{H}_{l}^{(2)}=\mathbf{H}_{l}^{(R D)} & , \text { if ACK } \\ L_{2}=L_{S D}, E_{2}=E_{S D}, \mathbf{H}_{l}^{(2)}=\mathbf{H}_{l}^{(S D)} & , \text { if NACK. }\end{cases}
$$

\section{JOINT TURBO EQUALIZATION}

Considering the second time slot as a set of $N$ virtual receive antennas, the two-time slot system (Source, Relay, and Destination) can be viewed as a point to point MIMO link with $M$ transmit and $2 N$ receive antennas, where the $2 N T \times 1$ block received signal vector $\mathbf{y}$ is constructed as,

$$
\underline{\mathbf{y}} \triangleq\left[\underline{\mathbf{y}}_{0}^{\top}, \cdots, \underline{\mathbf{y}}_{T-1}^{\top}\right]^{\top} \in \mathbb{C}^{2 N T}
$$

and

$$
\underline{\mathbf{y}}_{i} \triangleq\left[\begin{array}{c}
\mathbf{y}_{i}^{(1)} \\
\mathbf{y}_{i}^{(2)}
\end{array}\right] \in \mathbb{C}^{2 N}
$$

is the signal received over the $2 N$ virtual antennas. The block communication model corresponding to this two-slot scheme is given as,

$$
\underline{\mathbf{y}}=\underline{\mathcal{H}} \mathbf{s}+\underline{\mathbf{n}},
$$

where $\underline{\mathcal{H}} \in \mathbb{C}^{2 N T \times M T}$ is a block circulant matrix whose first $2 N T \times M$ column matrix is

$$
\begin{array}{r}
{\left[\underline{\mathbf{H}}_{0}^{\top}, \cdots, \underline{\mathbf{H}}_{L-1}^{\top}, \mathbf{0}_{M \times(T-L) 2 N}\right]^{\top}} \\
\text { with } L=\max \left(L_{S D}, L_{2}\right),
\end{array}
$$

and

$$
\underline{\mathbf{H}}_{l} \triangleq\left[\begin{array}{c}
\sqrt{E_{S D}} \mathbf{H}_{l}^{(S D)} \\
\sqrt{E_{2}} \mathbf{H}_{l}^{(2)}
\end{array}\right] \in \mathbb{C}^{2 N \times M}
$$

is the channel matrix correspoding to the $l$ th $(l=0, \cdots, L-1)$ virtual MIMO tap. Vector

$$
\underline{\mathbf{n}}=\left[\underline{\mathbf{n}}_{0}^{\top}, \cdots, \underline{\mathbf{n}}_{T-1}^{\top}\right]^{\top} \in \mathbb{C}^{2 N T}
$$

where

$$
\underline{\mathbf{n}}_{i} \triangleq\left[\begin{array}{c}
\mathbf{n}_{i}^{(1)} \\
\mathbf{n}_{i}^{(2)}
\end{array}\right] \sim \mathcal{N}\left(\mathbf{0}_{2 N \times 1}, \sigma^{2} \mathbf{I}_{2 N}\right)
$$

denotes the thermal noise of the two-slot equivalent MIMO system.

Note that the block circulant matrix $\underline{\mathcal{H}}$ can be block diagonalized in a Fourier basis. Therefore, applying the discrete fourier transform (DFT) on the two-slot received block signal vector (14) yields the following frequency domain block communication model,

$$
\underline{\mathbf{y}}_{f}=\underline{\boldsymbol{\Lambda}} \mathbf{s}_{f}+\underline{\mathbf{n}}_{f}
$$

where

$$
\underline{\mathcal{H}}=\mathbf{U}_{T, 2 N}^{\mathrm{H}} \underline{\boldsymbol{\Lambda}} \mathbf{U}_{T, M},
$$

with

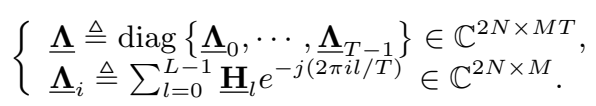

The proposed receiver iteratively performs (over slots) transmission combining and SISO decoding through the exchange of soft information in the form of Log-Likelihood Ratio (LLR) values between the joint (over slots) equalizer and the soft decoder. Let $\tilde{\mathbf{s}}$ denotes the conditional estimate of $\mathbf{s}$ and $\sigma_{t, i}^{2}$ denotes the conditional variance of $s_{t, i}$, the symbol at c.u $i$ transmitted over the $j$ th antenna. As presented in [14], the soft interferences cancellation and MMSE filtering can be implemented in the frequency domain using a forward and a backward filters. The MMSE estimate $\mathbf{z}_{f}$ on $\mathbf{s}_{f}$ can be expressed as,

$$
\mathbf{z}_{f}=\boldsymbol{\Phi} \underline{\mathbf{y}}_{f}-\mathbf{\Psi} \tilde{\mathbf{s}}_{f}
$$


where $\boldsymbol{\Phi}=\operatorname{diag}\left\{\boldsymbol{\Phi}_{0}, \cdots, \boldsymbol{\Phi}_{T-1}\right\}$ is the forward filter given by,

$$
\left\{\begin{array}{l}
\boldsymbol{\Phi}_{i} \triangleq \frac{1}{\sigma^{2}}\left\{\underline{\boldsymbol{\Lambda}}_{i}^{\mathrm{H}}-\mathbf{D}_{i} \mathbf{C}_{i}^{-1} \underline{\boldsymbol{\Lambda}}_{i}^{\mathrm{H}}\right\} \\
\mathbf{C}_{i}=\sigma^{2} \tilde{\boldsymbol{\Xi}}^{-1}+\mathbf{D}_{i}, \\
\mathbf{D}_{i}=\underline{\boldsymbol{\Lambda}}_{i}^{H} \underline{\boldsymbol{\Lambda}}_{i},
\end{array}\right.
$$

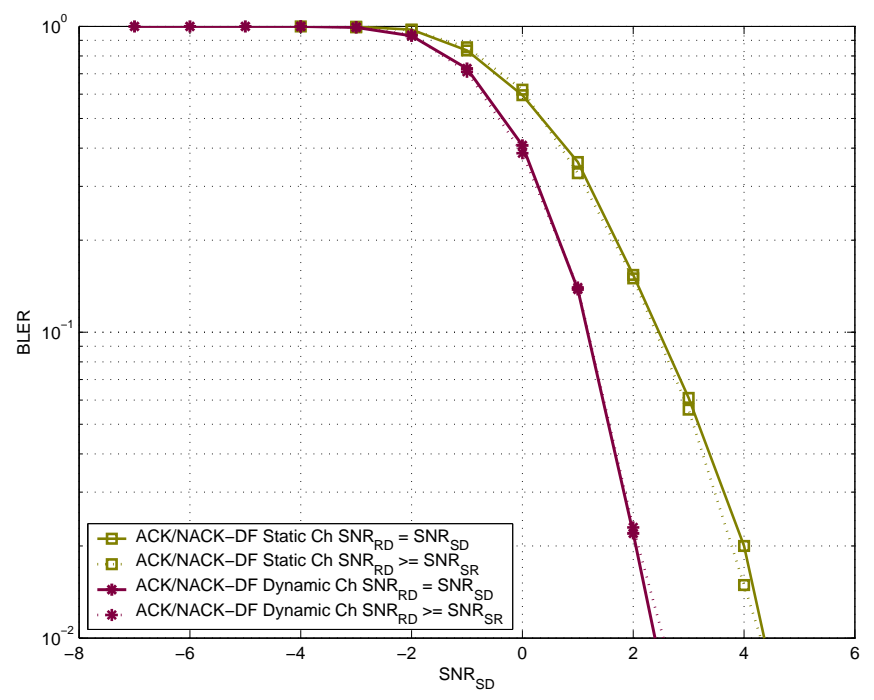

Figure 1: BLER versus $\mathrm{SNR}_{\mathrm{SD}}$ for $K=M=N=2$, all links have the same number of paths $L=3$ and $\mathrm{SNR}_{\mathrm{SD}}=\mathrm{SNR}_{\mathrm{SR}}$.

where $\tilde{\boldsymbol{\Xi}}$ is an unconditional covariance matrix computed as the time average of conditional covariance matrices $\boldsymbol{\Xi}_{i}$ defined as,

$$
\boldsymbol{\Xi}_{i} \triangleq \operatorname{diag}\left\{\sigma_{1, i}^{2}, \cdots, \sigma_{M, i}^{2}\right\},
$$

and $\boldsymbol{\Psi}=\operatorname{diag}\left\{\boldsymbol{\Psi}_{0}, \cdots, \boldsymbol{\Psi}_{T-1}\right\}$ is the backward filter given by,

$$
\left\{\begin{array}{l}
\mathbf{\Psi}_{i} \triangleq \boldsymbol{\Gamma}_{i}-\boldsymbol{\Upsilon} \\
\boldsymbol{\Gamma}_{i}=\frac{1}{\sigma^{2}}\left\{\mathbf{D}_{i}-\mathbf{D}_{i} \mathbf{C}_{i}^{-1} \mathbf{D}_{i}\right\} \\
\boldsymbol{\Upsilon}=\frac{1}{T} \sum_{i=0}^{T-1} \boldsymbol{\Gamma}_{i} .
\end{array}\right.
$$

After computing (24), the inverse DFT (IDFT) is then applied to $\mathbf{z}_{f}^{(k)}$ to obtain the equalized time domain sequence,

$$
\mathbf{z}=\mathbf{U}_{T, M}^{\mathrm{H}} \mathbf{z}_{f} .
$$

the MMSE estimate $z_{t, i}$ corresponding to antenna $t$ and c.u. $i$ can be simply extracted from $\mathbf{z}$ as $z_{t, i}=\mathbf{e}_{t, i}^{\mathrm{H}} \mathbf{z}$, with $\mathbf{e}_{t, i}$ denotes the $(M i+t)$ th vector of the canonical basis. The extrinsic LLRs value $\phi_{t, i, m}^{(e)}$ corresponding to coded and interleaved bit $b_{t, i, m}$ are then produced by the demapper using the max-log simplification,

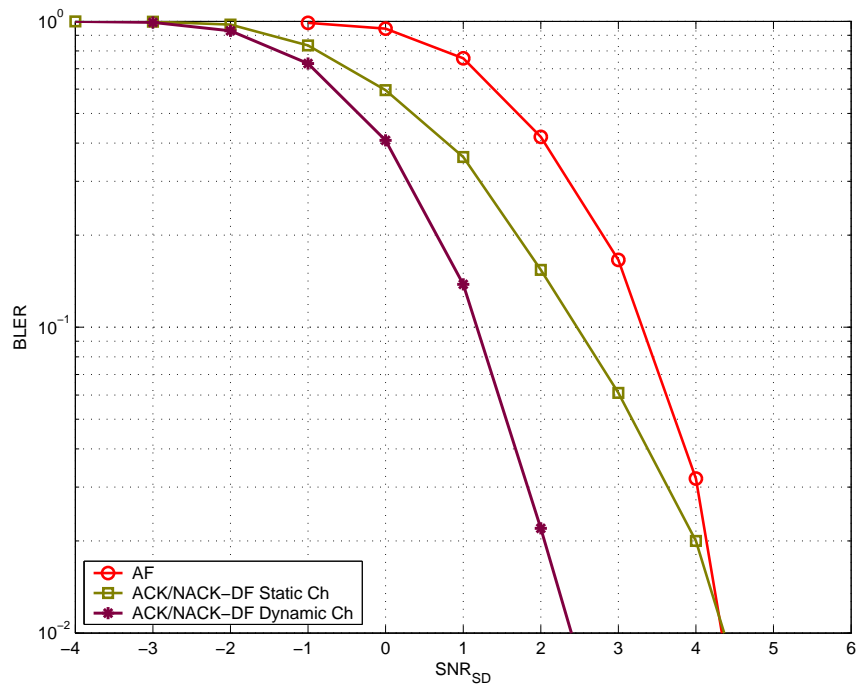

Figure 2: BLER versus $\mathrm{SNR}_{\mathrm{SD}}$ for $K=M=N=2$, all links have the same number of paths $L=3$ and $\mathrm{SNR}_{\mathrm{SD}}=\mathrm{SNR}_{\mathrm{SR}}=\mathrm{SNR}_{\mathrm{RD}}$.

$$
\begin{aligned}
\phi_{t, i, m}^{(e)} & =\min _{s \in \mathcal{S}_{0}^{m}}\left\{\frac{\left|z_{t, i}-g_{t, i} s\right|^{2}}{\theta_{t, i}^{2}}-\sum_{j \neq m} \phi_{t, i, j}^{(a)} \lambda_{j}\{s\}\right\} \\
& -\min _{s \in \mathcal{S}_{1}^{m}}\left\{\frac{\left|z_{t, i}-g_{t, i} s\right|^{2}}{\theta_{t, i}^{2}}-\sum_{j \neq m} \phi_{t, i, j}^{(a)} \lambda_{j}\{s\}\right\},
\end{aligned}
$$

where $g_{t, i}$ and $\theta_{t, i}$ denote, respectively, the equivalent channel gain at the output of equalizer and the residual interference variance corresponding to discrete time $j$, and transmit antenna $t . \phi_{t, i, j}^{(a)}$ is the a-priori LLR for coded bit $b_{t, i, j}$ obtained from the decoder in the previous iteration, $\lambda_{j}\{s\}$ is an operator extracting the $j$ th bit labeling the symbol $s \in \mathcal{S}$ and $\mathcal{S}_{\beta}^{m}$ is the set of symbols having the $m$ th bit set to $\beta$, i.e., $\mathcal{S}_{\beta}^{m}=\left\{s: \lambda_{m}\{s\}=\beta\right\}$. The LLRs calculated by the demapper are then de-interleaved and fed back to the softinput-soft-output (SISO) decoder.

\section{NUMERICAL RESULTS}

The system configuration used for performance evaluation has $K=M=N=2$, quadrature phase shift keying (QPSK) modulation, and a 16 state convolutional encoder with polynomial generators $(35,23)_{8}$. The length of the code frame is 2048 bits including tails. All links $(S \rightarrow D, S \rightarrow R$, $R \rightarrow D$ ) have the same frequency-selective fading channel profile, i.e., $L=3$ equal power paths. The $\mathrm{CP}$ length is $T_{C P}=3$. We use the Max-Log-MAP algorithm for SISO decoding. The iterative MMSE receiver at the destination node runs three turbo iterations. In all figures, AF denotes joint FDE-IC using the AF mode, while ACK/NACK-DF (Static $\mathrm{Ch}$ ) and ACK/NACK-DF (Dynamic Ch) denote the ACK/NACK-aided DF operating over a two and one-slot static fading $S \rightarrow D$ link, respectively.

First we consider a scenario where the $S \rightarrow D, S \rightarrow R$, $R \rightarrow D$ links are balanced. As expected, the DF mode outperforms the AF mode. However, when joint MMSE-FDE is 


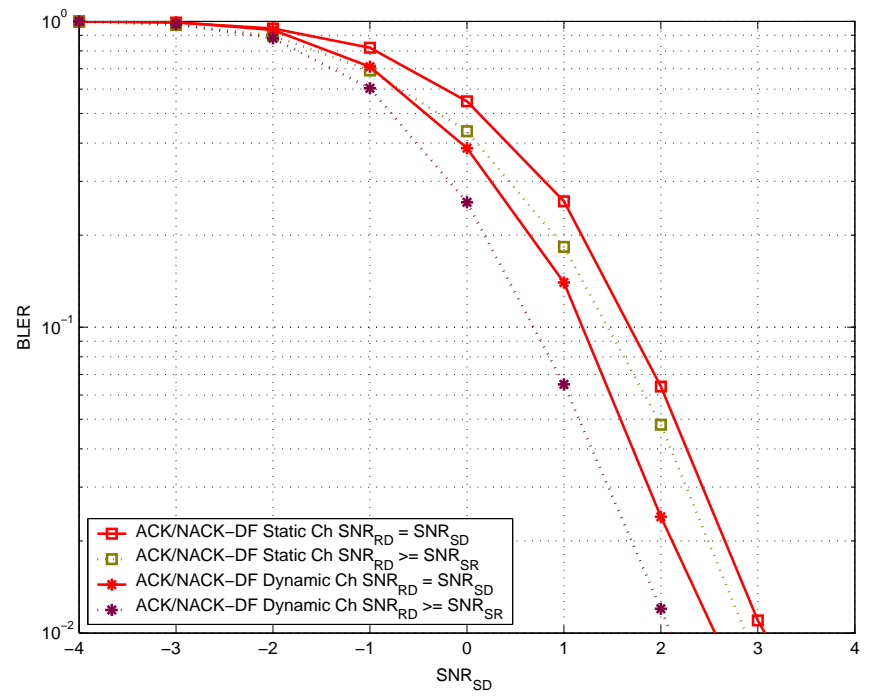

Figure 3: BLER versus $\mathrm{SNR}_{\mathrm{SD}}$ for $K=M=N=2$, all links have the same number of paths $L=3$ and $\mathrm{SNR}_{\mathrm{SR}}=\mathrm{SNR}_{\mathrm{SD}}+5 \mathrm{~dB}$.

able to collect the maximum achievable diversity gain (DF mode operating over one-slot static fading $S \rightarrow D$ link), the gap between the two modes is less than $2 \mathrm{~dB}$ at $10^{-2}$ BLER as it can be seen from Fig. 2. When the ACK/NACK-aided DF operates over a two-slot block static fading $S \rightarrow D$ link, the $\mathrm{AF}$ and $\mathrm{DF}$ modes have similar performance. For instance, the gap is only about $0.3 \mathrm{~dB}$ at $10^{-2}$ BLER. We also investigate the performance of the studied modes when the three links are unbalanced. From Fig. 1 and Fig. 3, we can see that the DF mode is relatively insensitive to the pathloss of the $R \rightarrow D$ link for $\mathrm{SNR}_{\mathrm{RD}} \geq \mathrm{SNR}_{\mathrm{SR}}$, while the AF mode is greatly affected (see Fig.4 and Fig. 5). Fig. 4 illustrates the performance of the studied modes when the $S \rightarrow D$ and $S \rightarrow R$ links are balanced and the $R \rightarrow D$ link experiences better quality (i.e., $\mathrm{SNR}_{\mathrm{RD}}=\mathrm{SNR}_{\mathrm{SD}}+5 \mathrm{~dB}$, $10 \mathrm{~dB}$ and $15 \mathrm{~dB})$. In the case of $\mathrm{DF}$ mode with no diversity gain, it is seen that the gap between the two modes is eliminated at $10^{-2}$ BLER. However, in the case of DF operating over a one-slot block static fading $S \rightarrow D$ link, the AF mode presents a performance degradation compared with the DF mode. For instance, the gap is about $1 \mathrm{~dB}$ at $10^{-2}$ BLER when SNR $_{\mathrm{SR}}=$ SNR $_{\mathrm{SD}}+15 \mathrm{~dB}$. Fig. 5 reports the performance of the studied modes when the $S \rightarrow R$ and $R \rightarrow D$ links experience better quality than the $S \rightarrow D$ link (i.e., $\mathrm{SNR}_{\mathrm{SR}}=\mathrm{SNR}_{\mathrm{SD}}+5 \mathrm{~dB}, \mathrm{SNR}_{\mathrm{RD}}=\mathrm{SNR}_{\mathrm{SD}}+5 \mathrm{~dB}$, $10 \mathrm{~dB}$, and $15 \mathrm{~dB})$. For this scenario, the AF mode clearly outperforms the DF mode with no diversity gain, and the gap reaches $1.1 \mathrm{~dB}$ for $\mathrm{SNR}_{\mathrm{SR}}=\mathrm{SNR}_{\mathrm{SD}}+15 \mathrm{~dB}$ at $10^{-2}$ BLER. Moreover, in the case of DF mode operating over a one-slot block static fading $S \rightarrow D$ link, the AF mode performs better when $\mathrm{SNR}_{\mathrm{SR}}=\mathrm{SNR}_{\mathrm{SD}}+10 \mathrm{~dB}$ and $\mathrm{SNR}_{\mathrm{SR}}=$ $\mathrm{SNR}_{\mathrm{SD}}+15 \mathrm{~dB}$. However, when $\mathrm{SNR}_{\mathrm{SR}} \leq \mathrm{SNR}_{\mathrm{SD}}+5 \mathrm{~dB}, \mathrm{AF}$ has degraded performance compared with $\mathrm{DF}$, and the gap is less than $2 \mathrm{~dB}$ at $10^{-2}$ BLER.

\section{CONCLUSION}

In this paper we have proposed a joint turbo equalizationaided combining scheme for both AF and ACK/NACK-aided

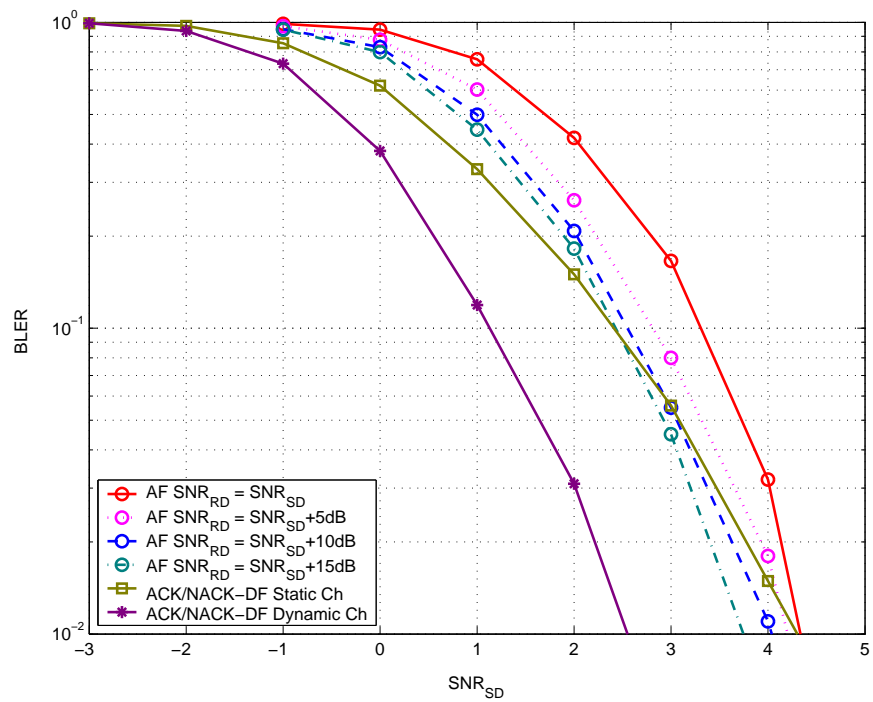

Figure 4: BLER versus $\mathrm{SNR}_{\mathrm{SD}}$ for $K=M=N=2$, all links have the same number of paths $L=3$ and $\mathrm{SNR}_{\mathrm{SD}}=\mathrm{SNR}_{\mathrm{SR}} \cdot \mathbf{A C K} / \mathrm{NACK}$-aided DF curves are still the same for $\mathrm{SNR}_{\mathrm{RD}}=\mathrm{SNR}_{\mathrm{SD}}+5 \mathrm{~dB}, 10 \mathrm{~dB}$ and $15 \mathrm{~dB}$

DF relaying protocols operating over a multiple-antenna frequency-selective fading relay channel. Simulation results have shown that both modes have similar performance and only a small gap is observed for some specific scenarios. As AF relaying presents less implementation requirements and computational complexity than DF, the proposed turbo combining scheme with AF is attractive for practical relay systems.

\section{REFERENCES}

[1] J. N. Laneman and G. W. Wornell, "Distributed space-time-coded protocols for exploiting cooperative diversity in wireless networks," IEEE Trans. Inform. Theory, vol. 49, no. 10, pp. 2415-2425, Oct. 2003.

[2] J. Laneman, D. Tse, and G. W. Wornell, "Cooperative diversity in wireless networks: Efficient protocols and outage behavior," IEEE Trans. Inform. Theory, vol. 50, no. 12, pp. 3062-3080, Dec. 2004.

[3] A. Sendonaris, E. Erkip, and B. Aazhang, "User cooperation diversity - Part I \& Part II," IEEE Trans. Commun., vol. 51, pp. 1927-1948, Nov. 2003.

[4] J. Boyer, D. D. Falconer, and H. Yanikomeroglu, "Multihop diversity in wireless relaying channels," IEEE Trans. on Comm., vol. 52, pp. 1820-1830, Oct. 2004.

[5] A. Adinoyi and H. Yanikomeroglu, "Multi-antenna aspects of parallel fixed wireless relays," IEEE WCNC, Las Vegas, Nevada, USA, April 2006.

[6] Z. Lin, E. Erkip, and A. Stefanov, "Cooperative regions for coded cooperative systems," IEEE GLOBECOM, Dallas, USA, Nov.-Dec. 2004.

[7] F.A. Onat, H. Yanikomeroglu, and S. Periyalwar, "Relay-assisted spatial multiplexing in wireless fixed relay networks,", IEEE GLOBECOM, San Francisco, USA, Nov.- Dec. 2006. 




Figure 5: BLER versus $\mathrm{SNR}_{\mathrm{SD}}$ for $K=M=N=2$, all links have the same number of paths $L=3$ and $\mathrm{SNR}_{\mathrm{SR}}=\mathrm{SNR}_{\mathrm{SD}}+5 \mathrm{~dB}$. ACK/NACK-aided DF curves are still the same for $\mathrm{SNR}_{\mathrm{RD}}=\mathrm{SNR}_{\mathrm{SD}}+5 \mathrm{~dB}, 10 \mathrm{~dB}$ and $15 \mathrm{~dB}$

[8] H. V. Khuong and T. Le-Ngoc, "A Bandwidth-efficient Cooperative Relaying Scheme with Limited Feedback Information" 24th Biennial Symposium on Communication 2008, pp. 175-178, June 2008.

[9] H. Mheidat and M. Uysal, "Equalization Techniques for Space-Time Coded Cooperative Systems", IEEE VTC-Fall, Los Angeles, California, USA, Sept. 2004.

[10] H. Mheidat, M. Uysal and N. Al-Dhahir,

"Single-Carrier Frequency Domain Equalization for Broadband Cooperative Communications," IEEE $W C N C$, Las Vegas, Nevada, USA, April 2006.

[11] H. Mheidat, M. Uysal, and N. Al-Dhahir, "Equalization Techniques for Distributed Space-Time Block Codes With Amplify-and-Forward Relaying," IEEE Trans. Signal Process., vol. 55, pp. 1839-1852, 2007.

[12] R. U.Nabar, F. W.Kneubiihler, and H. Boelcskei, "Performance Limits of Amplify-and-Forward Based Fading Relay channels,", IEEE ICASSP, Montreal, Canada, May 2004.

[13] Q. Jia, T. Lv and G. Ping, "An Efficient Scheme for Joint Equalization and Interference Cancellation in Distributed Cooperative Diversity Networks", CNSR, Halifax, Canada, May 2008.

[14] R. Visoz, A. O. Berthet, and S. Chtourou, "Frequency-domain block turbo-equalization for single-carrier transmission over MIMO broadband wireless channel," IEEE Trans. Commun., vol. 54, no. 12, pp. 2144-2149, Dec. 2006. 